HETEROCYCLES, Vol. 53, No. 4, 2000, pp. 831-839, Received, 3rd December, 1999

\title{
DIPOLAROPHILIC BEHAVIOR OF THE ISOXAZOLE RING IN INTRAMOLECULAR NITRILIMINE CYCLOADDITIONS
}

Gianluigi Broggini, ${ }^{\mathbf{b}}$ Luisa Garanti, ${ }^{\mathbf{a}}$ Giorgio Molteni, ${ }^{\mathbf{a}}$ and Gaetano Zecchi ${ }^{\mathbf{b}}$

a Dipartimento di Chimica Organica e Industriale dell'Università and Centro CNR, Via Golgi 19, 20133, Milano, Italy

${ }^{\mathbf{b}}$ Dipartimento di Scienze Chimiche, Fisiche e Matematiche, Università dell'Insubria, via Lucini 3, 22100 Como, Italy

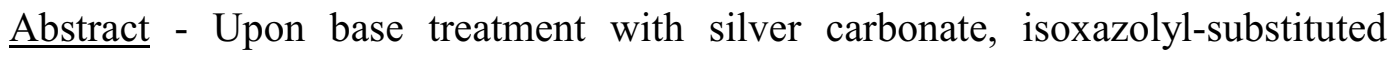
hydrazonoyl chlorides (4) and (12) undergo a sequential intra- intermolecular nitrilimine cycloaddition giving tetracyclic pyrazolydine derivatives (7) and (15).
\end{abstract}

Nitrilimine cycloadditions have been recognised as a fruitful tool in the construction of a variety of nitrogen-containing heterocycles, ${ }^{1-3}$ and a number of dipolarophilic functionalities, including heteroaromatic rings, ${ }^{4,5}$ have been employed for this purpose. Intramolecular versions of this methodology ${ }^{6}$ have been recently reported by us, in which the furan ${ }^{7}$ and thiophene ${ }^{8}$ rings behaves as dipolarophiles, giving rise to tricyclic pyrazolines. On pursuing our research line in this field, we present here the first example of intramolecular nitrilimine cycloaddition onto the isoxazole ring, whose dipolarophilic nature is still unexplored. It is worthwhile to anticipate that our intramolecular cycloaddition procedure allows the synthesis of the complex structures (TADP) (7) ${ }^{9}$ and (15), which are not easily accessible by other routes.

\section{RESULTS AND DISCUSSION}

Nitrilimines (4) were devised as the suitable substrates for the intramolecular cycloaddition onto the isoxazole ring. The building block for their synthesis was isoxazol-5-ylmethyl-3-oxobutanoate (2), which was readily prepared by reacting prop-2-ynyl 3-oxobutanoate $(\mathbf{1})^{10}$ with an equimolecular amount of 2,4,6-trimethyl-3,5-dichlorobenzonitrile oxide ${ }^{11}$ in refluxing carbon tetrachloride. Chlorination of 2 and subsequent treatment with the diazonium salt of the appropriate aniline gave the corresponding hydrazonoyl chlorides (4). The in situ generation of nitrilimines (5) was performed by treating a $0.02 \mathrm{M}$ 


\section{Scheme 1}

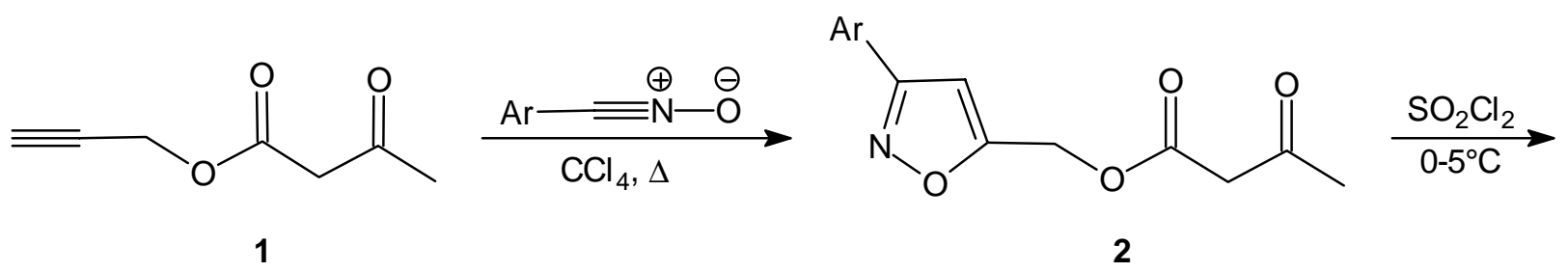<smiles>[X]c1ccc(NN=C(Cl)C(=O)OCc2cc([Al])no2)cc1</smiles><smiles></smiles>

5<smiles>Cc1cc(CO)on1</smiles><smiles>Cc1c(Cl)c([As])c(Cl)c([N+](=O)[O-])c1Cl</smiles>

a: $X=H ; b: X=M e ; c: X=C l$

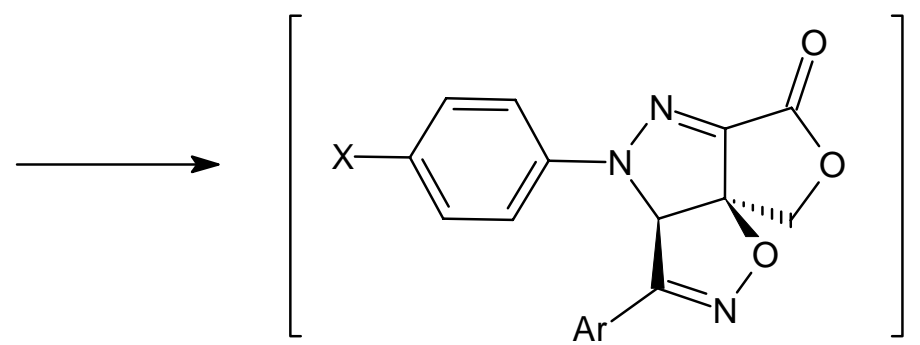

6

5<smiles>[X]c1ccc(N2[C@H]3C(Br)=NO[C@@]34COC(=O)[C@@]43N(c4ccc([X])cc4)N=C(C(=O)OCc4cc(Br)no4)N23)cc1</smiles> 
solution of 4 in dry dioxane at $75^{\circ} \mathrm{C}$. Reaction times, eluants and product yields are given in Table 1 , while analytical and spectroscopic data are collected in Tables 2 and 3.

Table 1. Reaction of hydrazonoyl chlorides (4) in the presence of silver carbonate.

\begin{tabular}{ccccc} 
Compd & $\begin{array}{c}\text { Time }^{\mathbf{a}} \\
(\mathrm{h})\end{array}$ & \multicolumn{2}{c}{ Products and yields $(\%)^{\mathbf{b}}$} & Eluent \\
& & $\mathbf{7}$ & $\mathbf{9}$ & \\
\hline $\mathbf{4 a}$ & 20 & 46 & 18 & $\operatorname{Et}_{2} \mathrm{O}-\operatorname{LP}^{\mathbf{d}}(1: 1)$ \\
$\mathbf{4 b}$ & 19 & 44 & 23 & $\operatorname{Et}_{2} \mathrm{O}-\operatorname{LP}^{\mathbf{d}}(1: 1)$ \\
$\mathbf{4 c}$ & 13 & 33 & 33 & $\operatorname{AcOEt}^{\mathbf{c} L P^{\mathbf{d}}}(1: 4)$ \\
\hline
\end{tabular}

${ }^{\mathbf{a}}$ In dry dioxane at $75^{\circ} \mathrm{C} .{ }^{\mathbf{b}}$ Isolation yields of pure products. ${ }^{\mathbf{c}}$ Silica gel column chromatography. ${ }^{\mathbf{d}} \mathrm{LP}=$ light petroleum bp $45-60^{\circ} \mathrm{C}$.

Structural assignements are unambiguous and rely upon analytical and spectral data, namely ${ }^{1} \mathrm{H}-\mathrm{NMR}$, MS and IR. ${ }^{1}$ H-NMR spectra of 7 are in fair agreement with those reported for related pyrazolo[4,5-d]isoxazoles. ${ }^{12}$ In addition, diagnostic evidence came from the IR spectra, where the absorption at $1800 \mathrm{~cm}^{-1}$ speaks in favor of unconjugated lactone carbonyl. ${ }^{13}$ The independent synthesis of 9 was carried out by reacting the corresponding nitrile oxide with 2-propyn-1-ol in boiling carbon tetrachloride.

The results depicted in Scheme 1 deserve some comments in order to rationalise the cycloaddition outcome. The final polycyclic products reasonably arise from the intramolecular cycloaddition of the transient nitrilimines (5) followed by an intermolecular cycloaddition of $\mathbf{5}$ onto the first-formed cycloadducts (6). Surprisingly, the above conditions precluded the isolation of the latter species. However, compounds (6) still contain two unsaturations, i.e. the $\mathrm{C}=\mathrm{N}$ bond of the isoxazolinic and the pyrazolinic rings, which are potentially susceptible to further dipolar attack. As a matter of fact, only the pyrazolinic $\mathrm{C}=\mathrm{N}$ bond of $\mathbf{6}$ behaves as dipolarophile in the intermolecular cycloaddition with $\mathbf{5}$, thus evidencing a fully site-selective process.

Due to the presence of the bulky 2,4,6-trimethyl-3,5-dichlorophenyl group, the exclusive cycloaddition onto the pyrazolinic double bond could be regarded as a consequence of steric factors. In order to verify this point, we synthesised the less hindered hydrazonoyl chloride (12), which was then submitted to the silver carbonate treatment (Scheme 2). Besides the formation of some quantity of 3-methyl-5hydroxymethylisoxazole (16), ${ }^{14}$ the only detectable cycloaddition product was again a TADP, namely 15. 
Table 2. Characterisation for compounds (7) and (9). ${ }^{\mathbf{a}}$

\begin{tabular}{|c|c|c|c|c|c|c|}
\hline \multirow{2}{*}{$\begin{array}{l}\text { Compd } \\
\text { (Formula) }\end{array}$} & \multirow{2}{*}{$\begin{array}{l}\mathrm{mp}^{\mathbf{b}} \\
\left({ }^{\circ} \mathrm{C}\right)\end{array}$} & \multirow{2}{*}{$\begin{array}{l}\text { IR (nujol) } \\
v\left(\mathrm{~cm}^{-1}\right)\end{array}$} & \multicolumn{3}{|c|}{ Microanalyses } & \multirow{2}{*}{$\begin{array}{l}\mathrm{MS} \\
m / z \\
\left(\mathrm{M}^{*}\right)\end{array}$} \\
\hline & & & $\begin{array}{c}\mathrm{C} \\
\text { found } \\
\text { calcd }\end{array}$ & $\begin{array}{c}\mathrm{H} \\
\text { found } \\
\text { calcd }\end{array}$ & $\begin{array}{c}\mathrm{N} \\
\text { found } \\
\text { calcd }\end{array}$ & \\
\hline $\begin{array}{l}7 \mathbf{a} \\
\left(\mathrm{C}_{42} \mathrm{H}_{34} \mathrm{~N}_{6} \mathrm{O}_{6} \mathrm{Cl}_{4}\right)\end{array}$ & 180 & 1800,1740 & $\begin{array}{l}58.79 \\
58.73\end{array}$ & $\begin{array}{l}4.04 \\
3.99\end{array}$ & $\begin{array}{l}9.86 \\
9.79\end{array}$ & 858 \\
\hline $\begin{array}{l}7 \mathbf{b} \\
\left(\mathrm{C}_{44} \mathrm{H}_{38} \mathrm{~N}_{6} \mathrm{O}_{6} \mathrm{Cl}_{4}\right)\end{array}$ & 213 & 1800,1735 & $\begin{array}{l}59.62 \\
59.58\end{array}$ & $\begin{array}{l}4.30 \\
4.32\end{array}$ & $\begin{array}{l}9.55 \\
9.48\end{array}$ & 886 \\
\hline $\begin{array}{l}7 \mathbf{c} \\
\left(\mathrm{C}_{42} \mathrm{H}_{32} \mathrm{~N}_{6} \mathrm{O}_{6} \mathrm{Cl}_{6}\right)\end{array}$ & 167 & 1800,1735 & $\begin{array}{l}54.37 \\
54.42\end{array}$ & $\begin{array}{l}3.51 \\
3.48\end{array}$ & $\begin{array}{l}9.11 \\
9.07\end{array}$ & 926 \\
\hline $\begin{array}{l}9 \\
\left(\mathrm{C}_{13} \mathrm{H}_{13} \mathrm{NO}_{2} \mathrm{Cl}_{2}\right)\end{array}$ & 147 & 3400 & $\begin{array}{l}54.77 \\
54.73\end{array}$ & $\begin{array}{l}4.62 \\
4.60\end{array}$ & $\begin{array}{l}4.83 \\
4.91\end{array}$ & 285 \\
\hline
\end{tabular}

${ }^{\mathbf{a}}$ NMR data are given in Table $3 .{ }^{\mathbf{b}}$ From diisopropyl ether.

Table 3. ${ }^{1} \mathrm{H}-\mathrm{NMR}$ data for compounds (7) and (9).

Compd $\quad{ }^{1} \mathrm{H}-\mathrm{NMR}\left(\mathrm{CDCl}_{3}\right)$

$\delta, J^{\mathbf{a}}$

$7 \mathbf{a}$

$2.18(6 \mathrm{H}, \mathrm{s}), 2.20(6 \mathrm{H}, \mathrm{s}), 2.58(6 \mathrm{H}, \mathrm{s}), 4.71(1 \mathrm{H}, \mathrm{d}, J=10.3), 5.00(1 \mathrm{H}, \mathrm{d}, J=10.3), 5.47$ $(1 \mathrm{H}, \mathrm{s}), 5.52(1 \mathrm{H}, \mathrm{d}, J=13.7), 5.64(1 \mathrm{H}, \mathrm{d}, J=13.7), 6.42(1 \mathrm{H}, \mathrm{s}), 6.70-7.20(10 \mathrm{H}, \mathrm{m})$

$7 \mathbf{b}$

$2.13(6 \mathrm{H}, \mathrm{s}), 2.20(6 \mathrm{H}, \mathrm{s}), 2.27(3 \mathrm{H}, \mathrm{s}), 2.32(3 \mathrm{H}, \mathrm{s}), 2.58(6 \mathrm{H}, \mathrm{s}), 4.68(1 \mathrm{H}, \mathrm{d}, J=10.2)$, $4.94(1 \mathrm{H}, \mathrm{d}, J=10.2), 5.42(1 \mathrm{H}, \mathrm{s}), 5.52(1 \mathrm{H}, \mathrm{d}, J=13.8), 5.63(1 \mathrm{H}, \mathrm{d}, J=13.8), 6.41(1 \mathrm{H}$, s), 6.55-7.17 (8H, m)

7c $\quad 2.16(6 \mathrm{H}, \mathrm{s}), 2.53(6 \mathrm{H}, \mathrm{s}), 2.59(6 \mathrm{H}, \mathrm{s}), 4.69(1 \mathrm{H}, \mathrm{d}, J=10.3), 4.98(1 \mathrm{H}, \mathrm{d}, J=10.3), 5.34$ $(1 \mathrm{H}, \mathrm{s}), 5.51(1 \mathrm{H}, \mathrm{d}, J=13.6), 5.61(1 \mathrm{H}, \mathrm{d}, J=13.6), 6.39(1 \mathrm{H}, \mathrm{s}), 6.56-7.20(8 \mathrm{H}, \mathrm{m})$

9

$2.14(6 \mathrm{H}, \mathrm{s}), 2.30(3 \mathrm{H}, \mathrm{s}), 2.54(1 \mathrm{H}$, br s $), 4.85(2 \mathrm{H}, \mathrm{s}), 6.15(1 \mathrm{H}, \mathrm{s})$

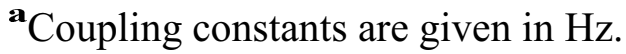




\section{Scheme 2}

$$
1+\mathrm{NO}_{2} \underset{\text { toluene, } 0^{\circ} \mathrm{C}}{\stackrel{\mathrm{PhNCO}_{2} \mathrm{Et}}{\longrightarrow}}
$$<smiles>CC(=O)C(Cl)C(=O)OCc1cc(C)no1</smiles><smiles>Cc1cc(COC(=O)/C(Cl)=N\N[Al])on1</smiles><smiles></smiles>

13<smiles>Cc1cc(CO)on1</smiles>

$16(28 \%)$<smiles>CC1=NO[C@]23COC(=O)C2=NN([Al])[C@H]13</smiles>

14<smiles>[Y4]c1cc(COC(=O)C2=NN([Al])[C@]34C(=O)OC[C@]35ON=C(C)[C@H]5N([Al])N4[C@H]2C)on1</smiles>

15 (48\%)<smiles>Clc1ccc(Br)cc1</smiles>

Therefore, it can be argued that the site-selectivity of the intermolecular cycloaddition is dictated by the electronic demands of the dipolarophilic sites of $\mathbf{6}$ and $\mathbf{1 4}$ rather than by steric encumbrance at the isoxazolinic $\mathrm{C}=\mathrm{N}$ bond.

As far the side-formation of compounds (9) and (16), one can note that they reflect a known degradative process of nitrilimines, which is illustrated in Scheme 3. Precedents of such behaviour pattern are available in the literature. ${ }^{15}$

\section{Scheme 3}

5 or 13
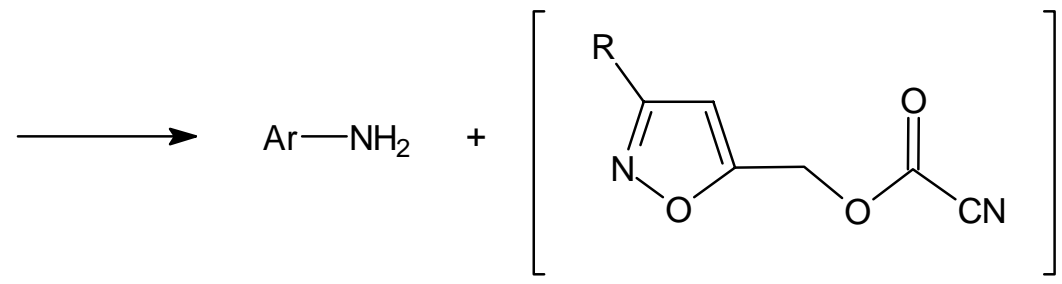
As a further stage of our work, we undertook a computational study at the AM1 level ${ }^{16}$ of nitrilimine (13) and cycloadduct (14). ${ }^{17}$ The results given in Figure 1 indicate that nitrilimine (13) should attack preferably the $\mathrm{C}=\mathrm{N}$ pyrazolinic bond rather than the isoxazolinic one by way of a $\mathrm{HOMO}_{\mathrm{dipole}}$ controlled process. This just accounts for experimental evidence.

13
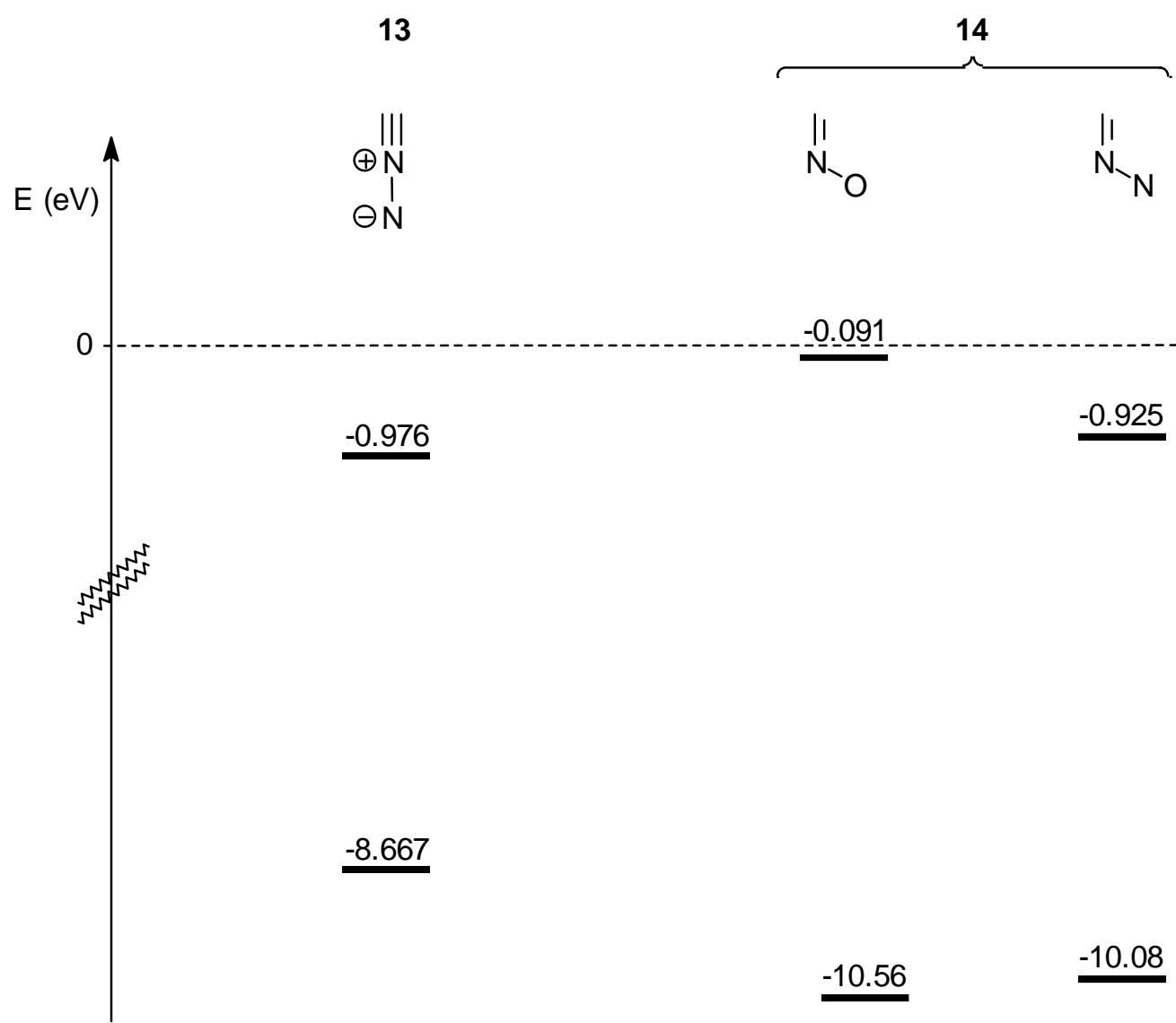

0.976

$-0.925$

Figure 1. FMO energies calculated with the AM1 method.

\section{EXPERIMENTAL}

Melting points were determined with a Büchi apparatus and are uncorrected. IR spectra were recorded on a FT IR Perkin Elmer $1725 \mathrm{X}$ spectrophotometer. MS spectra were determined with a VG-70EQ apparatus. ${ }^{1} \mathrm{H}-\mathrm{NMR}$ spectra were taken with a Bruker $\mathrm{AC} 300$ or $\mathrm{AMX} 300$ instrument in $\mathrm{CDCl}_{3}$ solutions; chemical shifts are given as ppm from $\mathrm{Me}_{4} \mathrm{Si}$ and $J$ values are given in $\mathrm{Hz}$.

[3-(2,4,6-Trimethyl-3,5-dichlorophenyl)isoxazol-5-yl]methyl 3-oxobutanoate (2). A solution of 1 (4.88 g, $34.8 \mathrm{mmol})$ and 2,4,6-trimethyl-3,5-dichlorobenzonitriloxide $(7.97 \mathrm{~g}, 34.8 \mathrm{mmol})$ in tetrachloromethane $(85 \mathrm{~mL})$ was refluxed for $6 \mathrm{~h}$. Evaporation of the solvent and subsequent crystallisation from diisopropyl ether gave 2; yield $11.69 \mathrm{~g}(91 \%) ; \mathrm{mp} 79^{\circ} \mathrm{C}$. IR (Nujol): 1750,1710 
$\left(\mathrm{cm}^{-1}\right) ;{ }^{1} \mathrm{H}$ NMR $\delta: 2.15(6 \mathrm{H}, \mathrm{s}), 2.30(3 \mathrm{H}, \mathrm{s}), 2.56(3 \mathrm{H}, \mathrm{s}), 3.58(2 \mathrm{H}, \mathrm{s}), 5.24(2 \mathrm{H}, \mathrm{s}), 6.29(1 \mathrm{H}, \mathrm{s})$; MS: $m / z 369\left(\mathrm{M}^{+}\right)$. Anal. Calcd for $\mathrm{C}_{17} \mathrm{H}_{17} \mathrm{NO}_{4} \mathrm{Cl}_{2}$ : C, 55.28; H, 4.64; N, 3.79. Found: C, 55.35; H, 4.61; N, 3.86 .

(3-Methylisoxazol-5-yl)methyl 3-oxobutanoate (10). A solution of 1 (4.88 g, 34.8 mmol), nitroethane $(3.26 \mathrm{~g}, 43.5 \mathrm{mmol})$ and triethylamine $(0.22 \mathrm{~g}, 2.20 \mathrm{mmol})$ in dry toluene $(80 \mathrm{~mL})$ was cooled to $0^{\circ} \mathrm{C}$. Phenyl isocyanate $(8.33 \mathrm{~g}, 70.0 \mathrm{mmol})$ was added dropwise under vigorous stirring and cooling at $0^{\circ} \mathrm{C}$. After $2 \mathrm{~h}$, the reaction mixture was allowed to warm to rt overnight. The solid material was filtered off, washed with toluene $(40 \mathrm{~mL})$ and the combined filtrates was evaporated giving $\mathbf{1 0}$ as undistillable oil not analytically pure; yield $5.76 \mathrm{~g}(84 \%)$. IR (Neat): 1740, $1710\left(\mathrm{~cm}^{-1}\right) ;{ }^{1} \mathrm{H}$ NMR $\delta: 2.23(3 \mathrm{H}, \mathrm{s}), 2.37(3 \mathrm{H}, \mathrm{s})$, $3.51(2 \mathrm{H}, \mathrm{s}), 5.19(2 \mathrm{H}, \mathrm{s}), 6.18(1 \mathrm{H}, \mathrm{s})$; MS: $m / z 197\left(\mathrm{M}^{+}\right)$.

\section{[3-(2,4,6-Trimethyl-3,5-dichlorophenyl)isoxazol-5-yl]methyl 2-chloro-3-oxobutanoate (3) and (3-} Methylisoxazol-5-yl)methyl 2-chloro-3-oxobutanoate (11); General Procedure. A solution of sulfuryl chloride (3.35 g, $25.0 \mathrm{mmol})$ in dry chloroform $(10.0 \mathrm{~mL})$ was slowly added $(1 \mathrm{~h})$ to a mixture of 2 or $\mathbf{1 0}$ $(25.0 \mathrm{mmol})$ and sodium hydrogencarbonate $(2.10 \mathrm{~g}, 25.0 \mathrm{mmol})$ in dry chloroform $(60 \mathrm{~mL})$, keeping the temperature in the range $0-5^{\circ} \mathrm{C}$. After $2 \mathrm{~h}$ at $\mathrm{rt}$, chloroform $(80 \mathrm{~mL})$ was added, and the mixture was washed with water $(2 \times 30 \mathrm{~mL})$. The organic layer was dried over sodium sulfate and the solvent was removed under reduced pressure giving $\mathbf{3}$ or $\mathbf{1 1}$ as undistillable oils not analytically pure.

(3); yield $9.77 \mathrm{~g}(97 \%)$. IR (Neat): 1760, $1715\left(\mathrm{~cm}^{-1}\right) ;{ }^{1} \mathrm{H}$ NMR $\delta: 2.15(6 \mathrm{H}, \mathrm{s}), 2.38(3 \mathrm{H}, \mathrm{s}), 2.57$ (3H, s), $4.87(1 \mathrm{H}, \mathrm{s}), 5.42(2 \mathrm{H}, \mathrm{s}), 6.30(1 \mathrm{H}, \mathrm{s})$; MS: $m / z 403\left(\mathrm{M}^{+}\right)$.

(11); yield $5.49 \mathrm{~g}(95 \%)$. IR (Neat): 1755, $1715\left(\mathrm{~cm}^{-1}\right) ;{ }^{1} \mathrm{H}$ NMR $\delta: 2.23(3 \mathrm{H}, \mathrm{s}), 2.38(3 \mathrm{H}, \mathrm{s}), 4.80(1 \mathrm{H}, \mathrm{s})$, $5.29(2 \mathrm{H}, \mathrm{s}), 6.21(1 \mathrm{H}, \mathrm{s})$; MS: $m / z 231\left(\mathrm{M}^{+}\right)$.

Hydrazonoyl chlorides (4) and (12); General Procedure. A solution of 3 or 11 (20.0 mmol) in cold methanol $(45 \mathrm{~mL})$ was added with sodium acetate $(2.72 \mathrm{~g}, 20.0 \mathrm{mmol})$. A cold aqueous solution of the appropriate arenediazonium chloride $(20.0 \mathrm{mmol})$ was added dropwise under vigorous stirring and icecooling. The mixture was allowed to stand overnight at $\mathrm{rt}$. The solven was partly removed under reduced pressure and the resulting mixture was extracted with ether $(2 \times 75 \mathrm{~mL})$. The organic layer was washed firstly with $5 \%$ aqueous sodium hydrogencarbonate $(50 \mathrm{~mL})$, then with water $(120 \mathrm{~mL})$, and dried over sodium sulfate. Evaporation of the solvent and subsequent crystallisation from diisopropyl ether gave the hydrazonoyl chlorides (4) or (12) in the pure state. 
(4a) yield $5.58 \mathrm{~g} \mathrm{(60 \% );} \mathrm{mp} 128^{\circ} \mathrm{C}$. IR (Nujol): 3260, $1720\left(\mathrm{~cm}^{-1}\right) ;{ }^{1} \mathrm{H}$ NMR $\delta: 2.16(6 \mathrm{H}, \mathrm{s}), 2.58(3 \mathrm{H}, \mathrm{s})$, $5.47(2 \mathrm{H}, \mathrm{s}), 6.34(1 \mathrm{H}, \mathrm{s}), 7.05-7.41(5 \mathrm{H}, \mathrm{m}), 8.45\left(1 \mathrm{H}\right.$, br s); MS: $m / z 465\left(\mathrm{M}^{+}\right)$. Anal. Calcd for $\mathrm{C}_{21} \mathrm{H}_{18} \mathrm{~N}_{3} \mathrm{O}_{3} \mathrm{Cl}_{3}$ : C, 54.19; H, 3.90; N, 9.03. Found: C, 54.12; H, 3.93 N, 9.10.

(4b) yield $5.56 \mathrm{~g}(58 \%) ; \mathrm{mp} 197^{\circ} \mathrm{C}$. IR (Nujol): 3270, $1720\left(\mathrm{~cm}^{-1}\right) ;{ }^{1} \mathrm{H}$ NMR $\delta: 2.17(6 \mathrm{H}, \mathrm{s}), 2.33(3 \mathrm{H}, \mathrm{s})$, $2.56(3 \mathrm{H}, \mathrm{s}), 5.56(2 \mathrm{H}, \mathrm{s}), 6.34(1 \mathrm{H}, \mathrm{s}), 7.11-7.29(4 \mathrm{H}, \mathrm{m}), 8.38\left(1 \mathrm{H}\right.$, br s); MS: $m / z 479\left(\mathrm{M}^{+}\right)$. Anal. Calcd for $\mathrm{C}_{22} \mathrm{H}_{20} \mathrm{~N}_{3} \mathrm{O}_{3} \mathrm{Cl}_{3}$ : C, 55.11; H, 4.21; N, 8.77. Found: C, 55.16; H, 4.25 N, 8.81.

(4c) yield $7.58 \mathrm{~g}(76 \%) ; \mathrm{mp} 127^{\circ} \mathrm{C}$. IR (Nujol): 3260, $1725\left(\mathrm{~cm}^{-1}\right) ;{ }^{1} \mathrm{H}$ NMR $\delta: 2.12(6 \mathrm{H}, \mathrm{s}), 2.58(3 \mathrm{H}, \mathrm{s})$, $5.52(2 \mathrm{H}, \mathrm{s}), 6.35(1 \mathrm{H}, \mathrm{s}), 7.13-7.35(5 \mathrm{H}, \mathrm{m}), 8.42(1 \mathrm{H}$, br s$)$; MS: $m / z 499\left(\mathrm{M}^{+}\right)$. Anal. Calcd for $\mathrm{C}_{21} \mathrm{H}_{17} \mathrm{~N}_{3} \mathrm{O}_{3} \mathrm{Cl}_{4}$ : C, 50.50; H, 3.43; N, 8.42. Found: C, 50.44; H, $3.40 \mathrm{~N}, 8.48$.

(12) yield $3.27 \mathrm{~g} \mathrm{(56 \% );} \mathrm{mp} \mathrm{89}{ }^{\circ} \mathrm{C}$. IR (Nujol): 3260, $1715\left(\mathrm{~cm}^{-1}\right) ;{ }^{1} \mathrm{H}$ NMR $\delta: 2.38(3 \mathrm{H}, \mathrm{s}), 5.44(2 \mathrm{H}, \mathrm{s})$, $6.23(1 \mathrm{H}, \mathrm{s}), 7.15-7.35(4 \mathrm{H}, \mathrm{m}), 8.50\left(1 \mathrm{H}\right.$, br s); MS: $m / z 292\left(\mathrm{M}^{+}\right)$. Anal. Calcd for $\mathrm{C}_{13} \mathrm{H}_{11} \mathrm{~N}_{3} \mathrm{O}_{3} \mathrm{Cl}_{2}: \mathrm{C}_{\text {, }}$ 53.42; H, 3.80; N, 14.38. Found: C, 53.36; H, 3.77 N, 14.44.

Silver carbonate treatment of hydrazonoyl chlorides (4) and (12); General Procedure. A solution of 4 or $12(10.0 \mathrm{mmol})$ in dry dioxane $(500 \mathrm{~mL})$ was treated with silver carbonate $(5.52 \mathrm{~g}, 20.0 \mathrm{mmol})$, and the mixture was stirred in the dark at $75^{\circ} \mathrm{C}$ for the time indicated in Table 1 (for compounds (4)) or $20 \mathrm{~h}$ (for compound (12)). The undissolved material was filtered off, the solvent was evaporated and then the residue was chromatographed on a silica gel column with the eluents given in Table 1 (for compounds (4)) or with ethyl acetate-hexane 1:1 (for compound (12)). Cycloadducts (7) or (15) was eluted firstly, followed by 9 or $16 .{ }^{14}$ Analytical and spectral data of compounds (7) and (9) are collected in Tables 2 and 3.

(15) yield $1.39 \mathrm{~g}(48 \%) ; \mathrm{mp} 98^{\circ} \mathrm{C}$. IR (Nujol): 1800, $1735\left(\mathrm{~cm}^{-1}\right) ;{ }^{1} \mathrm{H}$ NMR $\delta: 2.27(3 \mathrm{H}, \mathrm{s}), 2.38(3 \mathrm{H}, \mathrm{s})$, $4.60(1 \mathrm{H}, \mathrm{d}, J=10.2), 4.88(1 \mathrm{H}, \mathrm{d}, J=10.2), 5.48(1 \mathrm{H}, \mathrm{s}), 5.53(1 \mathrm{H}, \mathrm{d}, J=13.4), 5.59$ (1H, d, $J=13.4), 6.29$ $(1 \mathrm{H}, \mathrm{s}), 6.60-7.25(8 \mathrm{H}, \mathrm{m})$; MS: $m / z 582\left(\mathrm{M}^{+}\right)$. Anal. Calcd for $\mathrm{C}_{26} \mathrm{H}_{20} \mathrm{~N}_{6} \mathrm{O}_{6} \mathrm{Cl}_{2}: \mathrm{C}, 53.60 ; \mathrm{H}, 3.46 ; \mathrm{N}$, 14.43. Found: C, 53.66; H, $3.39 \mathrm{~N}, 14.50$.

3-[(2,4,6-Trimethyl-3,5-dichloro)phenyl]-5-hydroxymethylisoxazole (9). A solution of 2,4,6-trimethyl3,5-dichlorobenzonitriloxide $(0.50 \mathrm{~g}, 2.2 \mathrm{mmol})$ and 2-propyn-1-ol $(0.12 \mathrm{~g}, 2.2 \mathrm{mmol})$ in tetrachloromethane $(10 \mathrm{~mL})$ was refluxed for $6 \mathrm{~h}$. Evaporation of the solvent and subsequent crystallisation from diisopropyl ether gave 9; yield $0.53 \mathrm{~g}(85 \%)$.

\section{ACKNOWLEDGEMENTS}

We thank MURST and CNR for financial support. 


\section{REFERENCES}

1. P. Caramella and P. Grünanger, '1,3-Dipolar Cycloaddition Chemistry,' ed. by A. Padwa, WileyInterscience, NewYork, 1984, Vol. 1, Chapter 3.

2. P. A. Wade, 'Comprehensive Organic Synthesis,' Vol. 4, ed. by B. Trost and I. Fleming, Pergamon Press, New York, 1992, Chapter 4/10, pp. 1111-1168.

3. P. K. Claus, 'Methoden der Organische Chemie (Houben-Weil)', ed. by D. Klamann and H. Hagemann, Georg Thieme Verlag, Stuttgart, 1990, Band E14b, Teil 1, pp. 33-73.

4. G. Zecchi, Trends Heterocycl. Chem., 1991, $2,85$.

5. A. S. Shawali, Chem. Rev., 1993, 93, 2701.

6. G. Broggini, G. Molteni, and G. Zecchi, Heterocycles, 1998, 47, 541.

7. G. Broggini, G. Molteni, and G. Zecchi, J. Chem. Res. (S), 1998, 810.

8. G. Broggini, L. Garanti, G. Molteni, and G. Zecchi, J. Chem. Soc., Perkin Trans. 1, 1998, 4301.

9. According to the "replacement rule" nomenclature, the full name of compound (7a) should be: 4,7-bis(phenyl)-8-[(2,4,6-trimethyl-3,5-dichloro)phenyl]-3-oxo-7,7a-dihydro-4H-2,10-oxa-9-aza-4, 5,6a,7-tetraazadicyclopenta[a,h]pentalene-6-carboxilic acid 3-[(2,4,6-trimethyl-3,5dichloro)phenyl]isoxazol-5-ylmethyl ester. In order to avoid such awkward name, we propose the achronym TADP, standing for TetraAzaDicyclopentaPentalene for both compounds (7) and (15).

10. R. N. Lacey, J. Chem. Soc., 1954, 827.

11. P. Beltrame, C. Veglio, and M. Simonetta, J. Chem. Soc. (B), 1967, 867.

12. J. P. Gibert, C. Pétrus, and F. Pétrus, J. Heterocycl. Chem., 1977, 14, 253.

13. L. J. Bellamy, 'The Infra-red Spectra of Complex Molecules,' Methuen, London, 1958, Chapter 11.

14. A. Quilico and L. Panizzi, Gazz. Chim. Ital., 1939, 69, 536.

15. (a) N. H. Toubro and A. Holm, J. Amer. Chem. Soc., 1980, 102, 2093 (b) L. Garanti, G. Molteni, and G. Zecchi, Heterocycles, 1995, 40, 777 (c) L. Garanti, G. Molteni, and G. Zecchi, Heterocycles, 1997, 45, 1945.

16. As implemented in the Hyperchem package of programs.

17. M. J. S. Dewar, E. G. Zoebisch, E. F. Healy, and J. J. P. Stewart, J. Amer. Chem. Soc., 1985, ,107, 3902 . 